\title{
A Predictive Test of Outflow Enhancement by Ab Interno Trabeculectomy
}

Raoul Verma-Fuehring ${ }^{1}$, Mohamad Dakroub ${ }^{1}$, Hong Han ${ }^{1}$, Jost Hillenkamp ${ }^{1}$, Nils A. Loewen ${ }^{1}$

1. Department of Ophthalmology, University of Würzburg, Würzburg, Germany

\section{Correspondence to:}

Nils Loewen, MD, PhD

Department of Ophthalmology,

University Hospital Würzburg

Josef-Schneider-Straße 11

97080 Würzburg

Germany

loewen.nils@gmail.com 


\section{Abstract}

Purpose: To investigate trabeculopuncture (TP) for predicting the outcome of ab interno trabeculectomy (AIT). AIT is an effective, low-risk procedure for open angle glaucoma. Despite widespread utilization, it fails in patients with an unidentified distal outflow resistance.

Methods: We bisected 81 enucleated porcine eyes and perfused them for 72 hours. They were assigned to two groups: trial $(n=42)$ and control $(n=39)$. Intraocular pressure (IOP) was measured continuously. At 24 hours, four YAG-laser trabeculopunctures on the nasal trabecular meshwork were performed, followed by a $180^{\circ}$ AIT at the same site at 48 hours. Eyes were divided into TP and AIT responders and non-responders; the proportion of TP responders between both AIT groups was compared.

Results: Both post-TP and post-AIT IOPs were lower than baseline IOP ( $p=0.015$ and $p<0.01$, respectively). The success rates of TP and AIT were $69 \%$ and $85.7 \%$, respectively. The proportion of TP responders among AIT responders was greater than that of AIT non-responders $(p<0.01)$. Sensitivity and specificity values of TP as predictive test for AIT success were $77.7 \%$ and $83.3 \%$, respectively. The positive and negative predictive values were $96.6 \%$ and $38.5 \%$, respectively.

Conclusion: A 10\% reduction in IOP after TP can be used as predictor for the success (>20\% IOP decrease) of $180^{\circ} \mathrm{AIT}$ in porcine eyes.

Keywords: goniopuncture; ab interno trabeculectomy; porcine eyes; glaucoma; predictive test 


\section{Introduction}

Intraocular pressure (IOP) reduction is the only treatment for glaucoma ${ }^{1}$ demonstrated to be effective with high-quality level I evidence. ${ }^{2-4}$ Selective laser trabeculoplasty, now a recommended first line of treatment for most open angle glaucomas, ${ }^{5}$ and medications may achieve the desired IOP levels in many patients, but about 50\% still require surgery eventually. ${ }^{6}$ Traditional glaucoma filtering surgeries ${ }^{7}$ lower the IOP by bypassing the trabecular meshwork (TM) and draining the aqueous humor into a newly created epibulbar space ${ }^{8}$, but they are associated with a high rate of complications and require intensive postoperative care. ${ }^{9,10}$

Canal-based minimally invasive glaucoma surgeries produce far fewer complications and allow to intervene earlier ${ }^{11,12}$ because they lower the IOP by bypassing or removing the TM to enhance the physiological aqueous humor outflow route. ${ }^{11,13,14}$ Leading modalities are trabecular bypass stents $(\mathrm{TBS})^{15-17}$ and ab interno trabeculectomy (AIT) in which the TM is either ablated, ${ }^{18}$ incised, ${ }^{19}$ or excised. ${ }^{20}$ However, approximately $30 \%$ of patients experience an insufficient IOP reduction. ${ }^{21}$ One would expect the IOP to be close to the level of episcleral venous pressure, approximately $8 \mathrm{mmHg}$ but this pressure can rarely be achieved ${ }^{21}$ due to an increased post-trabecular resistance. ${ }^{22,23}$ So far, there is no presurgical test that could assess the post-trabecular resistance predict the outcome of AIT.

A noninvasive procedure that allows aqueous to bypass the proximal outflow resistance at least temporarily is ND:YAG laser-assisted trabeculopuncture (TP). Not unlike trabecular bypass stents, TP creates a focal opening through the trabecular meshwork (TM) and inner wall of Schlemm's canal (SC). ${ }^{24}$ In 1985, Epstein et al. investigated TP as a treatment for glaucoma but the subsequent IOP reduction was short-lived. ${ }^{24}$

Here, we hypothesized that TP could be used to assess the distal outflow tract function before AIT or TBS are considered. We deployed our porcine anterior chamber ex vivo perfusion model ${ }^{25,26}$ to develop a predictive test and address this unmet need. 


\section{Methods}

\section{Study Design}

In total, 81 hemisected, perfused porcine eyes were assigned to one of two groups: trial $(T)(n=42)$ and control $(C)(n=39)$. Eyes in the trial group underwent trabeculopuncture using a Nd:YAG laser 24 hours after incubation, followed by ab interno trabeculectomy a day later. The IOP was measured continuously for 72 hours, with baseline values being recorded 24 hours (IOP $\mathrm{BL}$ ) after the start of the experiment. Post-trabeculopuncture IOP (IOPTP) was measured 48 hours and post-ab interno trabeculectomy IOP (IOPAIT) at 72 hours.

Eyes in the control group did not undergo any procedures, but were incubated and monitored similarly for 72 hours.

\section{Preparation and Incubation}

Freshly enucleated porcine eyes were obtained from a local abattoir (Landschlachterei Issing, Retzbach, Bavaria, Germany) and processed within three hours postmortem. Institutional Animal Care and Use Committee review was waived because animals were not being sacrificed for the purpose of doing research. All eyes were stripped of extra-orbital tissue, placed in a $2.5 \%$ povidone-iodine solution for 30 seconds and rinsed with phosphate-buffered saline (PBS). After bisecting the eyes at the equator, the vitreous body, lens, and uvea were removed in one piece. The anterior segments were mounted in custom-made perfusion dishes, incubated at $37{ }^{\circ} \mathrm{C}$ and perfused with Dulbecco's Modified Eagle's Medium (DMEM) fortified with Penicillin/Streptomycin at a rate of $6 \mu \mathrm{l} / \mathrm{min}$ using a perfusion pump (Harvard PHD ULTRA ${ }^{\mathrm{TM}}$ CP Syringe Pump, Harvard Apparatus, Holliston, MA, USA). The dishes were connected to pressure transducers (Daltren DPT-200, Utah Medical Products Inc., Midvale, USA) which provided continuous IOP measurements at a rate of one reading every 2 minutes using LabChart (Version 8.1.16, ADInstruments, Sydney, Australia).

\section{Nd:YAG-Laser TP and AIT}

After perfusion for 24 hours, the anterior segments were removed from the dish and four evenly spaced trabeculopunctures were placed along the nasal $180^{\circ}$ of trabecular meshwork using a Qswitched Nd:YAG laser (VISUALS YAGIII, Zeiss, Oberkochen, Germany). Fifteen shots with an energy of 
7-10 mJ were applied for each puncture. A 180-degree ab interno trabeculectomy was performed 24 hours later along the same nasal $180^{\circ}$ of trabecular meshwork. ${ }^{26-28}$

\section{Canalograms}

After an anterior chamber exchange, eyes were placed under a stereomicroscope (Olympus SZX, Olympus K.K., Tokyo, Japan) for a baseline canalogram using a gravity-driven infusion of fluorescent microspheres (1:25 dilution, FluoSpheres $0.5 \mu \mathrm{m}$, Thermo Fisher Scientific Inc., Waltham, MA, USA) for 10 minutes. Canalograms were recorded and analyzed (cellSens Dimension, version 2.3, Olympus K.K., Tokyo, Japan) both after TP and AIT.

\section{Histology}

We obtained sagittal sections before TP as well as after TP and AIT and fixed them with 4\% paraformaldehyde in PBS for 24 hours. After rinsing them three times in PBS, they were embedded in paraffin, sectioned at 6-micron thickness, and stained with hematoxylin and eosin.

\section{Statistical Analysis}

Our sample size calculation indicated a minimum requirement of 35 eyes per group to achieve a testing power of 0.9. We analyzed data with SPSS Statistics (Version 26, IBM, New York, USA). Means and standard deviations were reported for all parameters. We tested for normal distribution with the Kolmogorov-Smirnov test and used a paired t-test or a Wilcoxon Signed Rank test to compare dependent means; an unpaired t-test or Mann-Whitney $U$ test was used for independent means. Oneway repeated measures multivariate analysis of variances (MANOVA) was used to compare more than two means. TP and AIT success were defined as a decrease of $5 \%$ and $10 \%$ from baseline IOP, respectively. We used the Fisher's exact test to compare the number of eyes that responded to TP and AIT to the number of eyes that responded to TP but failed AIT. Sensitivity, specificity, positive predictive value (PPV), and negative predictive value (NPV) were calculated. A receiver operating characteristic curve (ROC curve) was plotted for IOP reduction after TP and AIT success. For all tests, a p-value of 0.05 or less was considered statistically significant. 


\section{Results}

Pilot experiments with whole eyes showed that a transcorneal TP using a Ritch trabeculoplasty lens could not be accomplished reliably. Therefore, anterior segments were inverted and the TM to be lasered directly. The TM could be readily identified (Figure 1, BL1). The procedure resulted in small, well circumscribed pits of approximately $500 \mu \mathrm{m}$ in length and $250 \mu \mathrm{m}$ in depth (Figure 1, TP1). No obvious damage to adjacent tissue was detected upon inspection with a microscope. AlT removed the TM extensively, leading to a narrow continuous groove along the nasal quadrants (Figure 1, AIT1). The findings on histological sections (Figure 1, middle row) after both procedures corresponded well to our observations through an operating microscope. These were further supported by canalograms (Figure 1, BL3) which illustrated an improved localized outflow after TP (Figure 1, TP3) and further increased sectoral outflow in the nasal quadrants and in adjacent drainage segments after AIT (Figure 1, AIT3).

A total of 81 eyes were analyzed: 42 trial eyes (T) and 39 controls (C). Table 1 depicts the IOP parameters of both groups. Baseline IOP ( IOP $\left._{\mathrm{BL}}\right)$ was $16.4 \pm 4.5 \mathrm{mmHg}$ in T eyes and $15.2 \pm 3.9 \mathrm{mmHg}$ in $C$ eyes. There was no difference between both variables $(p=0.37)$. In the experimental group, mean $I_{\text {IOP }}$ and IOP lower than $\operatorname{IOP}_{\mathrm{BL}}(\mathrm{p}=0.02$ and $\mathrm{p}<0.001$, for IOP $\mathrm{TP}$ and IOPAIT, respectively). The three IOP measurements (IOP $\mathrm{BL}_{\mathrm{BL}} \mathrm{IOP}_{\mathrm{TP}}$, and IOP $\left.\mathrm{AIT}\right)$ were also found to be different from each other $(\mathrm{p}<0.001)$. Figure 2 illustrates the mean IOP levels of all 3 groups along with their respective average postprocedure IOP drops. Control eyes had an IOP that was $2.0 \pm 1.3 \mathrm{mmHg}$ higher at the end of the perfusion studies ( $p>0.05$ for both $24 \mathrm{~h}$ and $48 \mathrm{~h}$ ).

Table 2 depicts the distribution of TP responders in both AIT responders and AIT nonresponders. The total number of TP responders was 29 (69\%); for AIT, this value was 36 (85.7\%). The proportion of TP responders among AIT responders was greater than that among AIT non-responders (66.7\% vs $19 \%$, respectively, $p=0.007$ ). The positive and negative predictive values of TP as a test for predicting AIT success were $96.6 \%$ and $38.5 \%$, respectively. Sensitivity and specificity values were $77.7 \%$ and $83.3 \%$, respectively. This combination of values was plotted as an ROC curve of a $5 \%$ postTP IOP drop, predicting AIT success (Figure 3).

A subanalysis showed a mean IOPBL of $17.1 \pm 4.4 \mathrm{mmHg}$ and $11.9 \pm 2.7 \mathrm{mmHg}$ for AIT responders and AIT non-responders, respectively. These values differed significantly $(p=0.008)$. There was no 
difference in IOPTP and IOP responders, $76.9 \%(n=10)$ showed an IOP increase after TP of at least $10 \%$.

\section{Discussion}

In this paper, we used a porcine anterior segment model to assess the utility of TP in predicting the success of AIT. The lack of good predictive tests combined with the relatively high rate (30\%) of canalbased surgeries and implants led us to explore simple options to avoid unnecessary surgeries. Porcine eyes have been used as a model to study glaucoma extensively and were used here as well for that reason. ${ }^{29,30}$

We expected TP to cause an IOP drop in our porcine anterior segment perfusion model, similar to that reported in human eyes. TP is not unlike trabecular bypass stents, which also cause a focal opening in the TM, and increase outflow in our model. ${ }^{17}$ The amount of total energy used was higher than what is normally required in humans due to an at least three times thicker TM compared to human eyes. ${ }^{30}$ After AIT, a further decrease in IOP was seen because of the comprehensive excision of the nasal TM. Compared to a study by Dang et al., which tested outflow enhancements of three different AIT devices on porcine eyes, we found similar baseline IOP values $(16.35 \pm 4.52 \mathrm{mmHg}$ vs $15.93 \pm 2.08 \mathrm{mmHg})^{26}$ but observed a post-AIT IOP decrease by only $31 \%$, in contrast to $48 \%$ as reported in that study. ${ }^{26}$

The control group in our cohort experienced a small IOP increase by approximately $13 \%$ over 72 hours. This is in line with Dang et al. who observed a 10\% IOP increase in control eyes during 72 hours of incubation. ${ }^{31}$ To adjust for this, we chose $5 \%$ and $10 \%$ to be a satisfactory post-TP and postAIT IOP reduction, respectively. These values correspond to a $10 \%$ and $20 \%$ IOP reduction after each of these procedures. Clinically, a $20 \%$ post-AIT IOP reduction is often regarded as sufficient for mild to moderate glaucoma. ${ }^{32}$ Being able to predict this outcome will help avoid unnecessary procedures and decrease the burden on the healthcare system.

Interestingly, our subanalysis revealed that AIT responders had a higher baseline IOP compared to non-responders. This is perhaps to be expected, as AIT generally caused a greater decrease in eyes with higher baseline IOPs in clinical studies. ${ }^{33}$ AIT non-responders also had a higher mean IOP after TP, which did not reach significance with the number examined here. It is possible 
that the collapse of laser-induced trabeculopunctures temporarily decreases outflow facility as described before, ${ }^{34,35}$ which could similarly affect our non-TP responders if their TM was compromised in the area of TP and AIT. Additionally, there was no difference between baseline and post-AIT IOP levels in non-responders, which is indicative of a post-trabecular meshwork resistance in these eyes. These were not glaucomatous eyes, however. Ocular hypertension can be induced experimentally in pig eyes ${ }^{36}$ but does not occur in pigs naturally. We suspect that inadvertent compression of key elements of the distal outflow tract in the nasal quadrants by the compression ring of the perfusion mount is responsible for this. This would not necessarily lead to an increased IOP because at least 3/4th of the outflow tract has to be compromised ${ }^{37}$, but it can explain the failure to respond to TP and AIT. One has to be careful interpreting the results of this subanalysis, however, as there were only six AIT non-responders in our study.

A simple and noninvasive predictive test for canal-based surgeries that ablate, excise, disrupt or bypass the TM is urgently needed because of the rapidly increasing demand for these procedures. The implementation of $\mathrm{Nd}$ :YAG laser-TP for such a test is straightforward as this device is ubiquitously available in ophthalmology practices and clinics and most ophthalmologists are familiar with its use. ${ }^{38}$ Although the effect of TP is too short-lived to be useful for glaucoma treatment, ${ }^{39,40}$ it is precisely this benign nature that may afford a low-risk test of distal outflow resistance.

The positive predictive value (PPV) of $96.6 \%$ for TP as a test for AIT success is promising. Moreover, our sensitivity and specificity values of $77 \%$ and $83 \%$, respectively, are sufficient for a clinical test. Our data did not show a high negative predictive value (NPV, 38.5\%) in porcine eyes, however, because these are nonglaucomatous eyes. The NPV in human eyes should be higher, matching the AIT failure rate caused by a presumed higher rate of posttrabecular resistance than in pigs.

One limitation of our study is the ex-vivo setting. Hence, wound healing of the TM and its effect on IOP cannot be observed. Another limitation is the anatomical difference between porcine eyes and human eyes. In porcine eyes, the outflow tract consists of an angular aqueous plexus, whereas humans have a Schlemm's canal often with a single lumen. ${ }^{41,42}$ We used four evenly spaced TPs over the nasal angle not only to cover the extent of an AIT but also because of the decreased circumferential flow compared to a human Schlemm's canal. Clinically, reflux of blood from SC can normally be seen after a TP, a useful indicator of completion that is absent in an ex vivo model. We had to use the IOP decrease and an increased outflow of fluorescent beads as an indicator instead. 
In conclusion, a 10\% IOP reduction after trabeculopuncture can be used to predict a successful ab interno trabeculectomy in porcine eyes. 


\section{Acknowledgements: None}

Conflict of Interest: The authors of this paper declare that there are no conflicts of interest with regard to this publication.

\section{References}

1. Cohen LP, Pasquale LR. Clinical characteristics and current treatment of glaucoma. Cold Spring Harb Perspect Med. 2014;4(6). doi:10.1101/cshperspect.a017236 
2. Morton SC, Hassan Murad M, O'Connor E, et al. Quantitative Synthesis-An Update. Agency for Healthcare Research and Quality (US); 2018.

3. Berkman ND, Lohr KN, Ansari M, et al. Grading the Strength of a Body of Evidence When Assessing Health Care Interventions for the Effective Health Care Program of the Agency for Healthcare Research and Quality: An Update. Agency for Healthcare Research and Quality (US); 2013.

4. Burns PB, Rohrich RJ, Chung KC. The levels of evidence and their role in evidence-based medicine. Plast Reconstr Surg. 2011;128(1):305-310.

5. Gazzard G, Konstantakopoulou E, Garway-Heath D, et al. Selective laser trabeculoplasty versus eye drops for first-line treatment of ocular hypertension and glaucoma (LiGHT): a multicentre randomised controlled trial. Lancet. 2019;393(10180):1505-1516.

6. Jay JL, Murray SB. Early trabeculectomy versus conventional management in primary open angle glaucoma. Br J Ophthalmol. 1988;72(12):881-889.

7. Morgan WH, Yu D-Y. Surgical management of glaucoma: a review. Clin Experiment Ophthalmol. 2012;40(4):388-399.

8. Yu D-Y, Morgan WH, Sun X, et al. The critical role of the conjunctiva in glaucoma filtration surgery. Prog Retin Eye Res. 2009;28(5):303-328.

9. Jampel HD, Musch DC, Gillespie BW, et al. Perioperative complications of trabeculectomy in the collaborative initial glaucoma treatment study (CIGTS). Am J Ophthalmol. 2005;140(1):16-22.

10. Edmunds B, Thompson JR, Salmon JF, Wormald RP. The National Survey of Trabeculectomy. III. Early and late complications. Eye . 2002;16(3):297-303.

11. Ansari E. An Update on Implants for Minimally Invasive Glaucoma Surgery (MIGS). Ophthalmol Ther. 2017;6(2):233-241.

12. Richter GM, Coleman AL. Minimally invasive glaucoma surgery: current status and future prospects. Clin Ophthalmol. 2016;10:189-206.

13. Lee JY, Akiyama G, Saraswathy S, et al. Aqueous humour outflow imaging: seeing is believing. Eye . 2021;35(1):202-215.

14. Xin C, Wang H, Wang N. Minimally Invasive Glaucoma Surgery: What Do We Know? Where Should We Go? Transl Vis Sci Technol. 2020;9(5):15-15.

15. Esfandiari $\mathrm{H}$, Taubenslag $\mathrm{K}$, Shah $\mathrm{P}$, et al. Two-year data comparison of ab interno trabeculectomy and trabecular bypass stenting using exact matching. J Cataract Refract Surg. 2019;45(5):608-614.

16. Strzalkowska A, Strzalkowski P, Al Yousef Y, Grehn F, Hillenkamp J, Loewen NA. Exact matching of trabectome-mediated ab interno trabeculectomy to conventional trabeculectomy with mitomycin $\mathrm{C}$ followed for 2 years. Graefes Arch Clin Exp Ophthalmol. 2021;259(4):963-970.

17. Parikh HA, Loewen RT, Roy P, Schuman JS, Lathrop KL, Loewen NA. Differential Canalograms Detect Outflow Changes from Trabecular Micro-Bypass Stents and Ab Interno Trabeculectomy. Sci Rep. 2016;6:34705.

18. Minckler DS, Baerveldt G, Alfaro MR, Francis BA. Clinical results with the Trabectome for treatment of 
open-angle glaucoma. Ophthalmology. 2005;112(6):962-967.

19. Barkan O. GONIOTOMY FOR THE RELIEF OF CONGENITAL GLAUCOMA. Br J Ophthalmol. 1948;32(9):701728.

20. EIMallah MK, Seibold LK, Kahook MY, et al. 12-Month Retrospective Comparison of Kahook Dual Blade Excisional Goniotomy with Istent Trabecular Bypass Device Implantation in Glaucomatous Eyes at the Time of Cataract Surgery. Adv Ther. 2019;36(9):2515-2527.

21. Kaplowitz K, Bussel II, Honkanen R, Schuman JS, Loewen NA. Review and meta-analysis of ab-interno trabeculectomy outcomes. Br J Ophthalmol. 2016;100(5):594-600.

22. Chen S, Waxman S, Wang C, Atta S, Loewen R, Loewen NA. Dose-dependent effects of netarsudil, a Rhokinase inhibitor, on the distal outflow tract. Graefes Arch Clin Exp Ophthalmol. 2020;258(6):1211-1216.

23. Waxman S, Wang C, Dang Y, et al. Structure-Function Changes of the Porcine Distal Outflow Tract in Response to Nitric Oxide. Invest Ophthalmol Vis Sci. 2018;59(12):4886-4895.

24. Epstein DL, Melamed S, Puliafto CA, Steinert RF. Neodymium: YAG laser trabeculopuncture in open-angle glaucoma. Ophthalmology. 1985;92(7):931-937.

25. Loewen RT, Roy P, Park DB, et al. A Porcine Anterior Segment Perfusion and Transduction Model With Direct Visualization of the Trabecular Meshwork. Invest Ophthalmol Vis Sci. 2016;57(3):1338-1344.

26. Dang $Y$, Wang $C$, Shah $P$, et al. Outflow enhancement by three different ab interno trabeculectomy procedures in a porcine anterior segment model. Graefes Arch Clin Exp Ophthalmol. 2018;256(7):13051312.

27. Wang $\mathrm{C}$, Dang $\mathrm{Y}$, Shah $\mathrm{P}$, et al. Intraocular pressure reduction in a pigmentary glaucoma model by Goniotome Ab interno trabeculectomy. PLoS One. 2020;15(4):e0231360.

28. Wang C, Dang Y, Waxman S, Xia X, Weinreb RN, Loewen NA. Angle stability and outflow in dual blade ab interno trabeculectomy with active versus passive chamber management. PLoS One.

2017;12(5):e0177238.

29. Suarez T, Vecino E. Expression of endothelial leukocyte adhesion molecule 1 in the aqueous outflow pathway of porcine eyes with induced glaucoma. Mol Vis. 2006;12:1467-1472.

30. McMenamin PG, Steptoe RJ. Normal anatomy of the aqueous humour outflow system in the domestic pig eye. J Anat. 1991;178:65-77.

31. Dang $\mathrm{Y}$, Waxman $\mathrm{S}$, Wang $\mathrm{C}$, et al. Freeze-thaw decellularization of the trabecular meshwork in an ex vivo eye perfusion model. PeerJ. 2017;5:e3629.

32. Ahuja Y, Ma Khin Pyi S, Malihi M, Hodge DO, Sit AJ. Clinical results of ab interno trabeculotomy using the trabectome for open-angle glaucoma: the Mayo Clinic series in Rochester, Minnesota. Am J Ophthalmol. 2013;156(5):927-935.e2.

33. Loewen RT, Roy P, Parikh HA, Dang Y, Schuman JS, Loewen NA. Impact of a Glaucoma Severity Index on Results of Trabectome Surgery: Larger Pressure Reduction in More Severe Glaucoma. PLoS One. 2016;11(3):e0151926.

34. Rassow B, Witschel B. [Laser-trabecular puncture. Experimental studies]. Ophthalmologica. 
1975;170(4):362-369.

35. Ticho U, Cadet JC, Mahler J, Sekeles E, Bruchim A. Argon laser trabeculotomies in primates: evaluation by histological and perfusion studies. Invest Ophthalmol Vis Sci. 1978;17(7):667-674.

36. Dang $\mathrm{Y}$, Waxman $\mathrm{S}$, Wang $\mathrm{C}$, Loewen RT, Sun M, Loewen NA. A porcine ex vivo model of pigmentary glaucoma. Sci Rep. 2018;8(1):5468.

37. Hong Y, Wang C, Loewen R, et al. Outflow facility and extent of angle closure in a porcine model. Graefes Arch Clin Exp Ophthalmol. 2019;257(6):1239-1245.

38. Katsanos A, Tsaldari N, Gorgoli K, Lalos F, Stefaniotou M, Asproudis I. Safety and Efficacy of YAG Laser Vitreolysis for the Treatment of Vitreous Floaters: An Overview. Adv Ther. 2020;37(4):1319-1327.

39. Melamed S, Latina MA, Epstein DL. Neodymium:YAG laser trabeculopuncture in juvenile open-angle glaucoma. Ophthalmology. 1987;94(2):163-170.

40. Del Priore LV, Robin AL, Pollack IP. Long-term follow-up of neodymium: YAG laser angle surgery for openangle glaucoma. Ophthalmology. 1988;95(2):277-281.

41. Lei Y, Overby DR, Read AT, Stamer WD, Ethier CR. A new method for selection of angular aqueous plexus cells from porcine eyes: a model for Schlemm's canal endothelium. Invest Ophthalmol Vis Sci. 2010;51(11):5744-5750.

42. Loewen RT, Waxman S, Wang C, et al. 3D-Reconstruction of the human conventional outflow system by ribbon scanning confocal microscopy. PLoS One. 2020;15(5):e0232833. 


\section{Tables}

Table 1

Table 1 IOP parameters of trial and control group.

$1 \mathrm{IOP}_{\mathrm{BL}}=$ baseline IOP after 24 hours; IOP $\mathrm{TP}=\mathrm{IOP} 24$ hours after trabeculopuncture; IOP $\mathrm{AIT}=1 \mathrm{IOP} 24$ hours after ab interno trabeculectomy; IOP $48=1 \mathrm{IOP}$ after 48 hours of incubation; $\mathrm{IOP}_{72}=1 \mathrm{IOP}$ after 72 hours of incubation.

\begin{tabular}{|c|c|c|c|c|c|}
\hline Group & Parameter & $\begin{array}{l}\text { IOP-Value } \\
\text { (mmHg) }\end{array}$ & $\begin{array}{l}\text { Diff from } \\
\text { IOP }_{\mathrm{BL}} \\
\text { (mmHg) }\end{array}$ & $\begin{array}{l}\text { p-value for } \\
\text { IOP } \mathrm{P}_{\mathrm{BL}} \text { reduction }\end{array}$ & $\begin{array}{l}\text { p-value for } \\
\text { Trial vs. Control }\end{array}$ \\
\hline \multirow{3}{*}{$\begin{array}{c}\text { Trial } \\
(n=42)\end{array}$} & $1 O P_{B L}$ & $16.4 \pm 4.5$ & - & - & 0.37 \\
\hline & $\mathrm{IOP}_{\mathrm{TP}}$ & $14.6 \pm 4.3$ & $-1.7 \pm 3.8$ & $0.015^{*}$ & 0.16 \\
\hline & $\mathrm{IOP}_{\mathrm{AIT}}$ & $11.3 \pm 3.8$ & $-5.1 \pm 4.4$ & $<0.001^{*}$ & $<0.001^{*}$ \\
\hline \multirow{3}{*}{$\begin{array}{l}\text { Control } \\
(n=39)\end{array}$} & $\mathrm{IOP}_{\mathrm{BL}}$ & $15.2 \pm 3.9$ & - & - & 0.37 \\
\hline & $1 \mathrm{IP}_{48}$ & $15.9 \pm 4.0$ & $0.8 \pm 1.6$ & $0.01 *$ & 0.16 \\
\hline & $1 \mathrm{OP}_{72}$ & $17.3 \pm 4.6$ & $2.0 \pm 1.3$ & $<0.001^{*}$ & $<0.001^{*}$ \\
\hline
\end{tabular}




\section{Table 2}

Table 2 Distribution of AIT and TP responders and non-responders. AIT = ab interno trabeculectomy. TP = YAG-assisted Trabeculopuncture

\begin{tabular}{|c|c|c|c|}
\hline Outcome & $\begin{array}{c}\text { AIT responders } \\
\text { (n, \%) }\end{array}$ & $\begin{array}{c}\text { AlT non-responders } \\
\text { (n, \%) }\end{array}$ & Total (n, \%) \\
\hline $\begin{array}{c}\text { TP responders } \\
\text { (n, \%) }\end{array}$ & $28(66.7 \%)$ & $1(2.4 \%)$ & $29(69.0 \%)$ \\
\hline $\begin{array}{c}\text { TP non-responders } \\
(\mathbf{n}, \%)\end{array}$ & $8(19.0 \%)$ & $5(11.9 \%)$ & $13(31.0 \%)$ \\
\hline Total $\mathbf{( n )}$ & $36(85.7 \%)$ & $6(14.3 \%)$ & $42(100 \%)$ \\
\hline
\end{tabular}


Table 3

Table 3 Subanalysis in AIT Success of the trial group. Negative values represent an increase in IOP. $\mathrm{AIT}=\mathrm{ab}$ interno trabeculectomy; IOP $\mathrm{BL}=$ baseline IOP; IOP $\mathrm{IOP}_{\mathrm{AIT}}=\mathrm{IOP} 24$ hours after ab interno trabeculectomy

\begin{tabular}{|c|c|c|c|c|}
\hline \multirow{2}{*}{ Group } & Parameter & $\begin{array}{c}\text { IOP reduction } \\
\text { (mmHg) }\end{array}$ & $\begin{array}{c}\text { IOP-Value } \\
(\mathbf{m m H g})\end{array}$ & $\begin{array}{c}\text { p-value for reduction } \\
\text { from IOP }\end{array}$ \\
\hline \multirow{2}{*}{$\begin{array}{c}\text { AIT } \\
\text { responders }\end{array}$} & $\mathrm{IOP}$ & $17.1 \pm 4.4$ & \\
\cline { 2 - 5 } & $\mathrm{IOP}$ & & $14.8 \pm 4.5$ & $0.002^{*}$ \\
\cline { 2 - 5 } & $\mathrm{IOP}_{\mathrm{AIT}}$ & 6.0 & $11.1 \pm 3.9$ & $<0.001^{*}$ \\
\hline \multirow{2}{*}{$\begin{array}{c}\text { AIT non- } \\
\text { responders }\end{array}$} & $\mathrm{IOP}_{\mathrm{BL}}$ & & $11.9 \pm 2.7$ & 0.53 \\
\cline { 2 - 5 } & $\mathrm{IOP}_{\mathrm{TP}}$ & -1.5 & $13.5 \pm 3.4$ & 1.0 \\
\cline { 2 - 5 } & $\mathrm{IOP}_{\mathrm{AIT}}$ & -0.4 & $12.4 \pm 2.6$ & \\
\hline
\end{tabular}




\section{Figures}

Fig. 1 Visualization of the effects of trabeculopuncture (TP) and ab interno trabeculectomy (AIT)

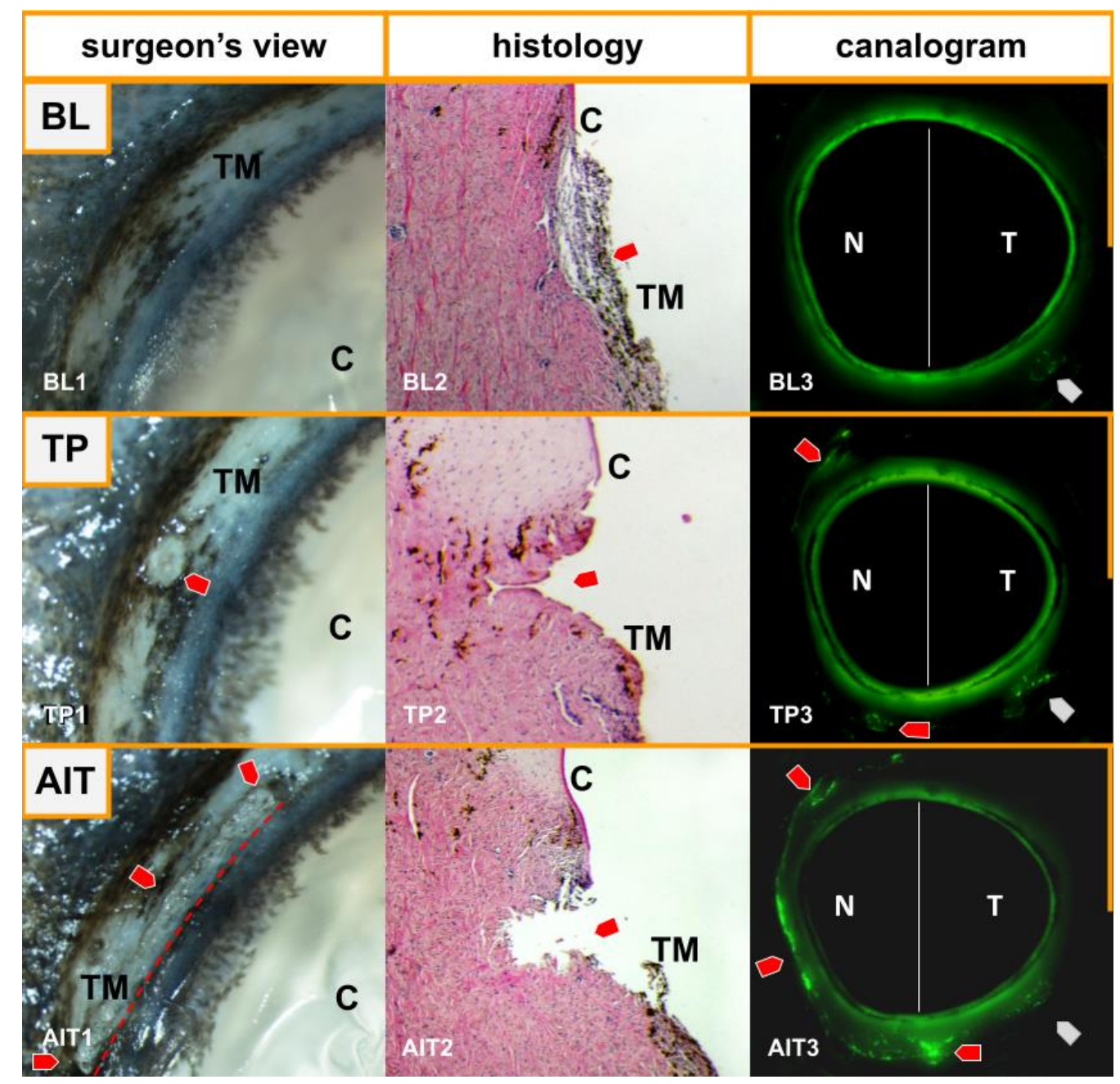

Fig. 1 Visualization of the effects of trabeculopuncture (TP) and ab interno trabeculectomy (AIT). Microscopic images, histology sections, and canalograms at baseline (BL), after trabeculopuncture (TP), and after ab interno trabeculectomy (AIT). BL1: Microscopic image of the porcine trabecular meshwork at baseline. BL2: Histological analysis of a section of the intact porcine TM (red arrow). BL3: Canalogram image of an anterior segment perfused with fluorescent spheres. No sphere collection can be in the nasal episcleral veins at baseline. Single beads were detected in the temporal region (gray arrow). TP1: Ex vivo image of a nasal YAG-TP. TP2: Histological analysis of a section after TP on the TM (red arrow). TP3: Canalogram image of an anterior segment perfused with fluorescent spheres after TP. Fluorescent spheres can now be visualized in the nasal episcleral veins (red arrows). Temporal fluorescence remained unchanged from baseline (gray arrow). AIT1: Image post trabeculectomy. The red arrows outline the excision in the TM. AIT2: Histological section after AIT. Trabecular meshwork is excised (red arrow). AIT3: Canalogram image of an anterior segment perfused with fluorescent spheres. An increased accumulation of spheres along the nasal circumference can be seen (red arrows). $\mathrm{TM}=$ trabecular meshwork; $\mathrm{C}=$ cornea; $\mathrm{N}=$ nasal; $\mathrm{T}=$ temporal. 
Fig. 2 Intraocular pressure (IOP) levels at baseline and post intervention

\section{IOP}

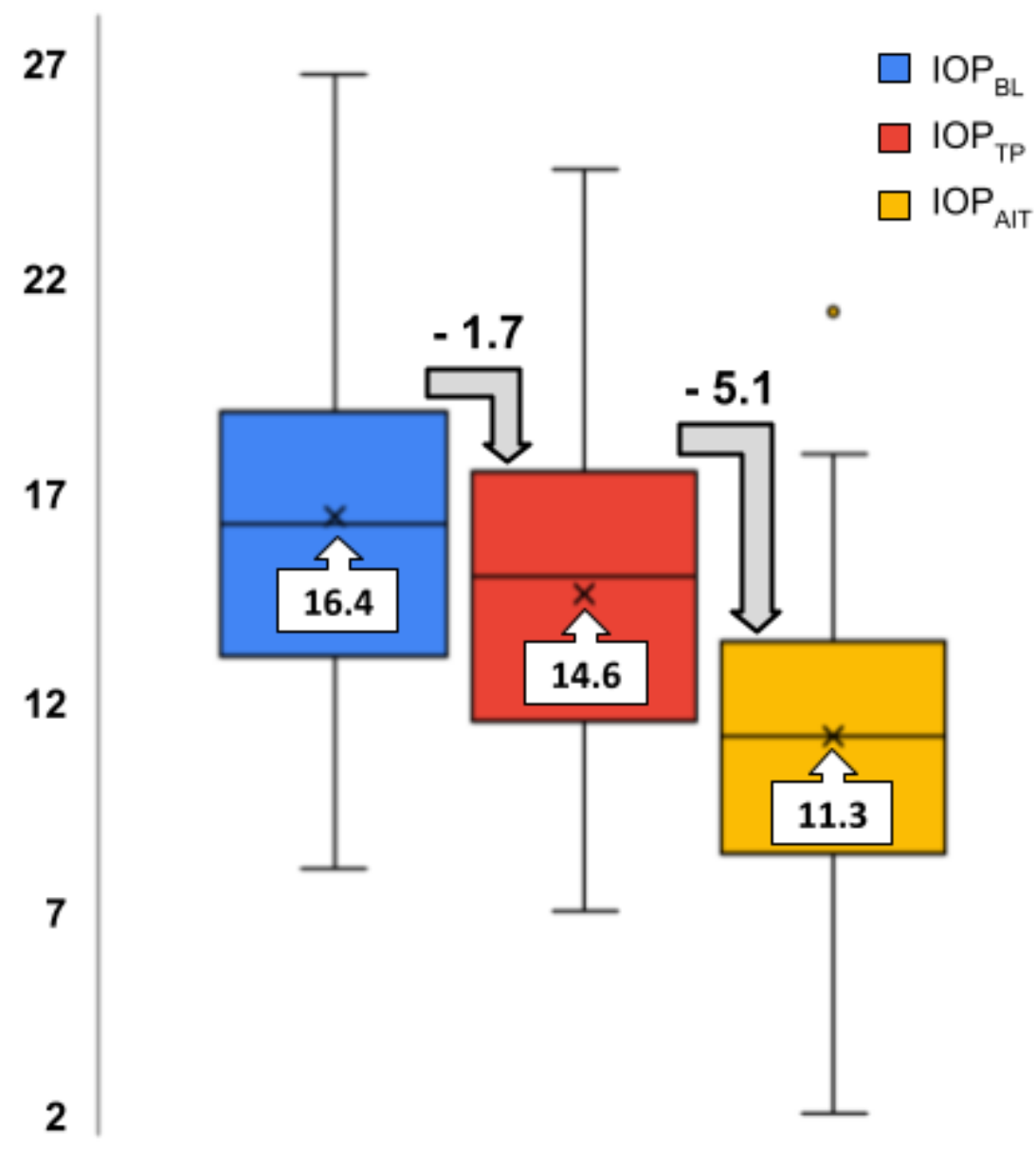

Fig. 2 Boxplots for mean IOP levels at Baseline (IOP $\mathrm{BL}_{\mathrm{BL}}$ ), post trabeculopuncture (IOP $\left.\mathrm{TP}\right)$, and post ab interno trabeculectomy (IOPAIT). The figures above the grey arrows correspond to the mean IOP reduction after trabeculopuncture and ab interno trabeculectomy, respectively. 
Fig 3 Receiver Operating Characteristics Curve

\section{ROC Curve}

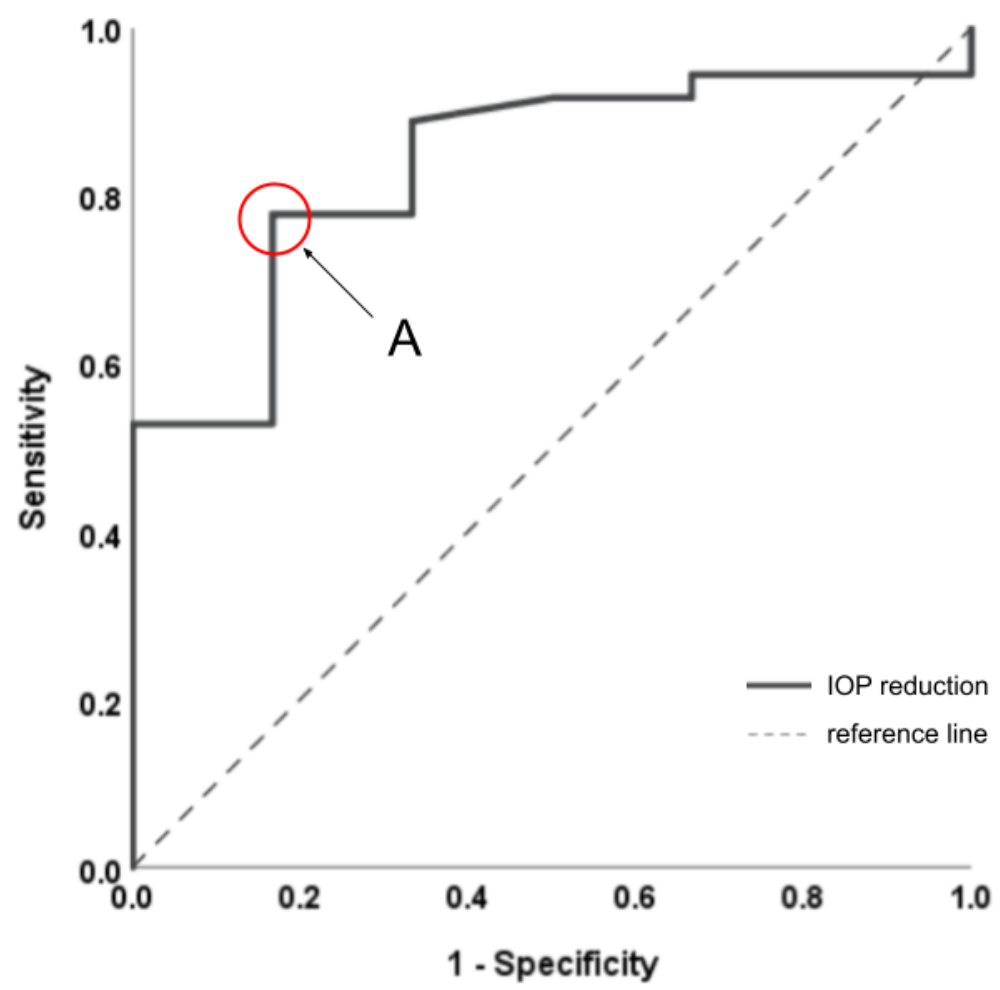

Fig. 3 Receiver operating characteristics (ROC) curve for post-trabeculopuncture IOP reduction in detecting ab interno trabeculectomy responders. Point A corresponds to a 5\% IOP drop (resembling a $10 \%$ IOP reduction) after trabeculopuncture representing a sensitivity of $76.4 \%$ and specificity of $83.3 \%$. 